\title{
Avaliação da integralidade na atenção primária à saúde da criança na perspectiva dos cuidadores
}

\author{
Assessment of comprehensiveness in children's primary health care as seen by caregivers \\ Evaluación de la integralidad en la atención primaria de salud infantil según la perspectiva de los cuidadores
}

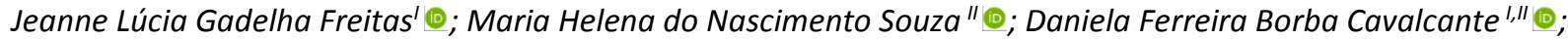

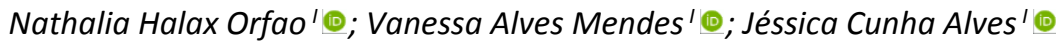 \\ 'Universidade Federal de Rondônia, Porto Velho, RO, Brasil; "Universidade Federal do Rio de Janeiro, Rio de Janeiro, RJ, Brasil
}

\begin{abstract}
RESUMO
Objetivo: avaliar, sob a ótica do cuidador, o atributo da integralidade na atenção primária à saúde da criança no município de Porto Velho, Brasil. Método: estudo avaliativo, transversal realizado com 420 cuidadores de crianças atendidas em um hospital infantil em 2017. Utilizou-se o Primary Care Assessment Tool Brasil - versão criança e os dados foram analisados pelo software Statistic 13.0. A pesquisa foi aprovada pelo Comitê de Ética e Pesquisa. Resultados: na avaliação das dimensões do atributo integralidade, o escore médio dos serviços disponíveis $(4,67)$ e dos serviços prestados $(5,26)$ à criança mostrou baixa orientação para a atenção primária à saúde, principalmente no que tange às orientações recebidas pelos profissionais, sobre crescimento, segurança, benefícios sociais, problemas visuais e de comportamento da criança. Conclusão: o atributo integralidade na saúde da criança está presente, porém de forma fragmentada, necessitando que os serviços revejam as prioridades nos cuidados à criança junto ao familiar/cuidador.
\end{abstract}

Descritores: Saúde da Criança; Integralidade em Saúde; Atenção Primária à Saúde; Avaliação em saúde.

\section{ABSTRACT}

Objective: to assess, from the caregiver's perspective, the comprehensiveness of children's primary health care in Porto Velho, Brazil. Method: in this evaluative, cross-sectional study of 420 caregivers at a children's hospital in 2017, the Primary Care Assessment Tool Brazil - children's version was used and data were analyzed using Statistic 13.0 software. The research was approved by a research ethics committee. Results: in evaluation of dimensions of comprehensiveness, children's services available scored an average of 4.67 and services provided, 5.26, showing poor orientation towards primary health care, particularly as regards the guidance received by health personnel on children's growth, safety, social benefits, eyesight and behavioral problems. Conclusion: comprehensive child health care is present, although fragmented, requiring that services review child care priorities with family members/caregivers.

Descriptors: Child Health; Integrality in Health; Primary Health Care; Health assessment.

\section{RESUMEN}

Objetivo: evaluar, desde la perspectiva del cuidador, la integralidad de la atención primaria de salud infantil en Porto Velho, Brasil. Método: en este estudio evaluativo, transversal de 420 cuidadores en un hospital infantil en 2017, se utilizó la Herramienta de Evaluación de Atención Primaria Brasil - versión infantil y los datos se analizaron mediante el software Estadística 13.0. La investigación fue aprobada por un comité de ética en investigación. Resultados: en la evaluación de las dimensiones de integralidad, los servicios disponibles para la infancia obtuvieron un promedio de 4,67 y los servicios prestados, 5,26 , mostrando una mala orientación hacia la atención primaria de salud, particularmente en lo que respecta a la orientación que recibe el personal de salud sobre el crecimiento, la seguridad, los beneficios sociales, la vista del niño y problemas de comportamiento. Conclusión: la atención integral de la salud infantil está presente, aunque fragmentada, lo que requiere que los servicios revisen las prioridades del cuidado infantil con los miembros de la familia / cuidadores.

Descriptores: Salud del Niño; Integralidad en Salud; Atención Primaria de Salud; Evaluación de la salud.

\section{INTRODUÇÃO}

A Atenção Primária à Saúde (APS) é caracterizada por um conjunto de ações, no âmbito individual e coletivo, que abrange a promoção e a proteção da saúde, prevenção de agravos, diagnóstico, tratamento, reabilitação, redução de danos e a manutenção da saúde. Tem o objetivo de desenvolver uma atenção integral que impacte nos determinantes e condicionantes de saúde além de promover a autonomia das pessoas ${ }^{1,2}$.

Considerada o primeiro nível assistencial do sistema de saúde, a APS é responsável pela integralidade da atenção, além de atuar como coordenadora da Rede de Atenção à Saúde (RAS) centralizada na família, orientação e participação comunitária ${ }^{1,2}$. Estudos internacionais mostram que a qualidade de uma APS está diretamente associada a melhores resultados de saúde ${ }^{3,4}$.

Apoio técnico e financeiro: Fundação de Amparoà Pesquisa do Estado de Rondônia (FAPERO), Programa Pesquisa para o SUS (PPSUS) - Chamada FAPERO/MS-DECIT/CNPq/SESAU-RO-n003/2016. Autora correspondente: Daniela Ferreira Borba Cavalcante. E-mail: danielaferreiraborba@yahoo.com.br

Editora responsável: Sergio Corrêa Marques 
No Brasil, o desempenho dos serviços da APS tem sido avaliado por meio de investigações sobre a extensão e presença de atributos essenciais que qualificam tais serviços, como a acessibilidade, longitudinalidade, coordenação do cuidado e a integralidade ${ }^{1,2,5,6}$, que constituiu o foco deste estudo. $O$ atributo integralidade na APS é entendido como um conjunto de ações preventivas e curativas prestadas no âmbito individual e coletivo por uma mesma unidade de saúde em articulação com serviços de outros níveis de atenção, com a finalidade de oferecer respostas satisfatórias às demandas de cuidado da população e superar a fragmentação da assistência. Nessa perspectiva, a partir dos serviços disponíveis e prestados às crianças e aos seus familiares pode ser evidenciado uma atenção integral de caráter biopsicossocial, mediante ações de promoção, prevenção, cura e reabilitação adequadas ${ }^{2,5,6}$.

Entretanto, embora o atributo da integralidade seja considerado essencial para o funcionamento de uma unidade de APS, alguns estudos apontam que na ótica dos cuidadores há fragilidades no tocante a problemas relacionados à estrutura dos serviços e insuficiência de orientações fornecidas pelos profissionais de saúde acerca do cuidado infantil ${ }^{5-}$ ${ }^{9}$. Consequentemente há necessidade de elaboração de planos de cuidados que contemplem o contexto do usuário e atendam suas reais necessidades de saúde ${ }^{10}$

Com a crescente expansão do modelo de atenção primária à saúde num país de dimensões continentais como o Brasil, faz-se cada vez mais necessário a realização de estudos voltados para a avaliação da qualidade dos serviços de saúde oferecidos ${ }^{1,10-12}$. E nestas investigações, a visão das pessoas atendidas é essencial para subsidiar a identificação de problemas e a reorientação do modelo assistencial com vistas à melhoria da qualidade desses serviços e da oferta de um cuidado de saúde integral ${ }^{5-7,9-13}$.

Não obstante, verifica-se que a maioria dos estudos avaliativos na APS evidenciam os obstáculos que a população enfrenta referente à acessibilidade aos serviços ${ }^{3-4,7-13}$ e que ainda são incipientes as pesquisas voltadas para a avaliação dos serviços disponíveis e prestados pelas equipes de saúde da família sobretudo no atendimento à criança, sob a perspectiva do familiar cuidador ${ }^{5,6,8}$.

Diante do exposto, este estudo objetivou avaliar, sob a ótica do cuidador, o atributo da integralidade na atenção primária à saúde da criança no município de Porto Velho, Brasil.

\section{MÉTODO}

Estudo avaliativo transversal realizado no município de Porto Velho, estado de Rondônia, situado na região norte do Brasil. O município é a maior região de saúde do estado e conta com $63 \%$ de cobertura da Estratégia Saúde da Família (ESF). Em 2017, o município possuía um Índice de Desenvolvimento Humano de 0,736 e população de 519.436 habitantes, sendo 51.292 crianças menores de cinco anos, ou seja, 9,8\% da população residente nesse município ${ }^{14}$.

O cenário do estudo foi o Hospital e Pronto Socorro Infantil Cosme e Damião (HICD) considerado como único serviço público de referência em urgência e emergência infantil da capital. Recebe também a chamada demanda espontânea das unidades de saúde da capital e do interior. Em 2015, das 56.370 crianças atendidas $73 \%$ correspondeu à demanda de baixa e média complexidade ${ }^{15}$.

A população foi constituída de familiares/cuidadores responsáveis pelas crianças menores de cinco anos, atendidas no referido hospital. O cálculo amostral teve como base o total de 2.483 crianças menores de cinco anos, atendidas em 2015. Realizou-se o cálculo por meio do EPI INFO 3.5.4 ${ }^{16}$, considerando erro de $5 \%$, intervalo de confiança de $95 \%$. A amostra inicial prevista foi de 333 participantes, contudo, considerando-se um acréscimo de $20 \%$ para perdas e recusas, obteve-se uma amostra final de 420 familiares/cuidadores.

Considerou-se como critérios de inclusão, cuidadores de crianças residentes no município, com idade igual ou superior a 18 anos, que estivessem de posse da Caderneta de Saúde da Criança no momento da entrevista, que declararam possuir cadastrados em uma das 22 unidades de saúde da família do município de Porto Velho. Foram excluídos os cuidadores de crianças que se encontravam em unidade de internação ou de tratamento intensivo e aqueles que possuíam problemas se saúde mental ou deficiências que impossibilitasse a compreensão do instrumento.

A coleta dos dados ocorreu no período de 01 de abril a 30 de outubro de 2017. Foi realizado previamente o teste piloto do instrumento e capacitação de três pesquisadores que foram supervisionados pela coordenadora da pesquisa. As entrevistas aconteceram no período matutino e vespertino de segunda a sexta-feira, em local reservado situado na recepção do referido hospital onde os responsáveis das crianças aguardavam atendimento. Após o convite para participar e a explicação sobre objetivo, os responsáveis que aceitaram foram convidados a assinar o Termo de Consentimento Livre e Esclarecido. O tempo médio de cada entrevista foi cerca de 15 minutos.

O instrumento utilizado na coleta de dados foi o questionário Primary Care Assessment Tool (PCATool) versão criança, desenvolvido por Barbara Starfield para avaliar os atributos da APS nos princípios de saúde propostos por Donabedian². Este instrumento foi validado/adaptado à realidade brasileira, submetido à tradução, tradução reversa, adaptação, 
debriefing e validação de conteúdo/constructo e de confiabilidade ${ }^{17}$. O questionário é composto por 55 itens subdivididos em 10 componentes. Neste estudo, foi avaliado o atributo integralidade e seus dois componentes, serviços disponíveis ( 9 itens) e serviços prestados (5 itens). As respostas de cada item do componente serviço disponível abrangem: "com certeza sim" (valor =4); "provavelmente sim" (valor = 3); "provavelmente não" (valor = 2); "com certeza não" (valor = 1) e "não sei/não lembro" (valor $=9)^{10}$. As respostas de cada item do componente serviços prestados abrangem: "com certeza sim" (valor =4); "provavelmente sim" (valor = 3); "provavelmente não" (valor = 2); "com certeza não" (valor = 1) e "não sei/não lembro" (valor =9). Ao final, a soma dos escores atribuídos em cada componente, resulta na classificação forte $(\geq 6,6)$ ou baixa $(<6,6)$ orientação para APS ${ }^{18}$.

Após preenchimento, cada questionário foi imediatamente digitalizado em dupla entrada em planilha Excel por dois entrevistadores simultaneamente. Foram descartados 15 questionários em função do elevado percentual de respostas do tipo "não sei/não lembro" que ficou superior à soma dos itens avaliados relativos ao componente serviços disponíveis.

Os dados foram analisados por meio de estatística descritiva no software Statistic versão 13.0, a partir da média aritmética simples para definição dos escores. Ressalta-se que o escore de cada variável e componente foi transformado para uma escala de 0 a 10 seguindo a fórmula: escore ajustado $=($ Escore obtido -1$) \times 10 / 3$. Posteriormente, houve uma categorização a partir dos parâmetros estabelecidos para forte $(\geq 6,6)$ ou baixa $(<6,6)$ orientação para APS ${ }^{18}$.

O estudo fez parte do projeto matriz "Avaliação da Atenção à Saúde da Criança em Porto Velho - RO" do Centro de Estudos e Pesquisa em Saúde Coletiva. A pesquisa cumpriu exigências da Resolução 466/12 ${ }^{19}$ sendo aprovada no parecer número 1.849.757 pelo Comitê de Ética em Pesquisa da Universidade Federal de Rondônia.

\section{RESULTADOS}

$\mathrm{Na}$ ótica dos cuidadores, os componentes serviços disponíveis $(4,67)$ e prestados $(5,26)$ do atributo integralidade tiveram baixa orientação para a APS considerando o escore esperado $(\geq 6,66)$.

O componente serviços disponíveis (SD) do atributo integralidade na avaliação do familiar/cuidador da criança é apresentado na Figura 1.

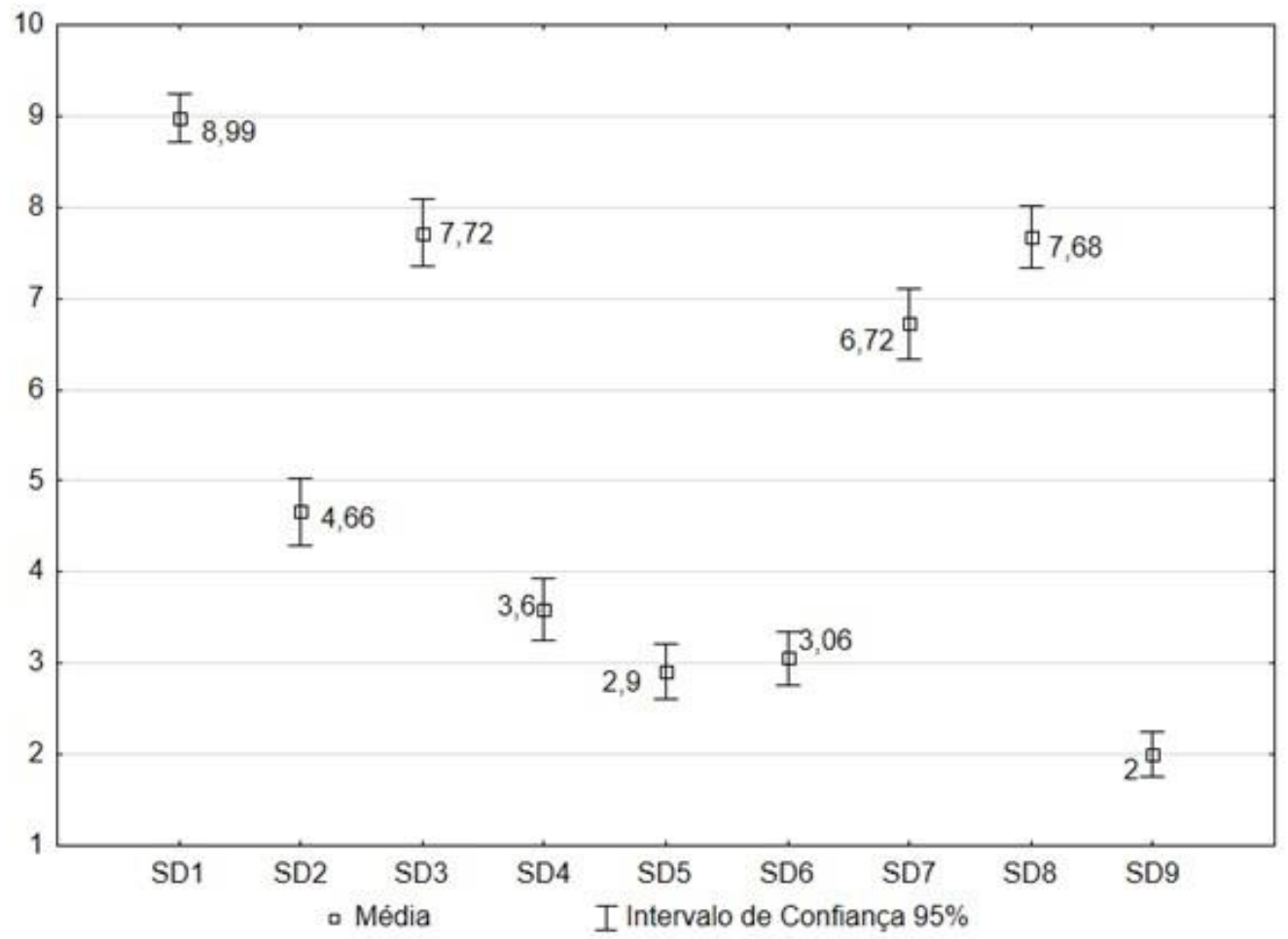

Legenda:SD - Serviços Disponíveis; SD1 - Vacinas (imunizações); SD2-Disponibilidade para verificar se a família pode participar de algum programa de assistência social ou benefícios sociais; SD3 - Planejamento familiar ou métodos anticoncepcionais; SD4 - Programa de suplementação nutricional (ex.: leite e alimentos); SD5 - Aconselhamento ou tratamento para o uso prejudicial de drogas; SD6 - Aconselhamento para problemas de saúde mental; SD7 - Sutura de um corte que necessite de pontos; SD8 Aconselhamento e solicitação de teste anti-HIV; SD9 - Identificação de problemas visuais.

FIGURA 1: Média e intervalo de confiança do componente serviços disponíveis do atributo integralidade na avaliação do familiar/cuidador da criança, Porto Velho, Rondônia, 2017. 
Dos nove itens avaliados no componente serviços disponíveis, cinco foram classificados como baixa orientação para APS, sendo estes relacionados à disponibilidade para verificar se a família pode participar de algum programa de assistência social ou benefícios sociais $(4,66)$, programa de suplementação nutricional $(3,60)$, aconselhamento ou tratamento para o uso prejudicial de drogas $(2,90)$, aconselhamento para problemas de saúde mental $(3,06)$ e identificação de problemas visuais $(2,0)$. Ainda nesse componente o item melhor avaliado foi referente à oferta de vacinas.

Já o componente serviços prestados do atributo integralidade na avaliação do familiar/cuidador da criança é demonstrado na Figura 2.

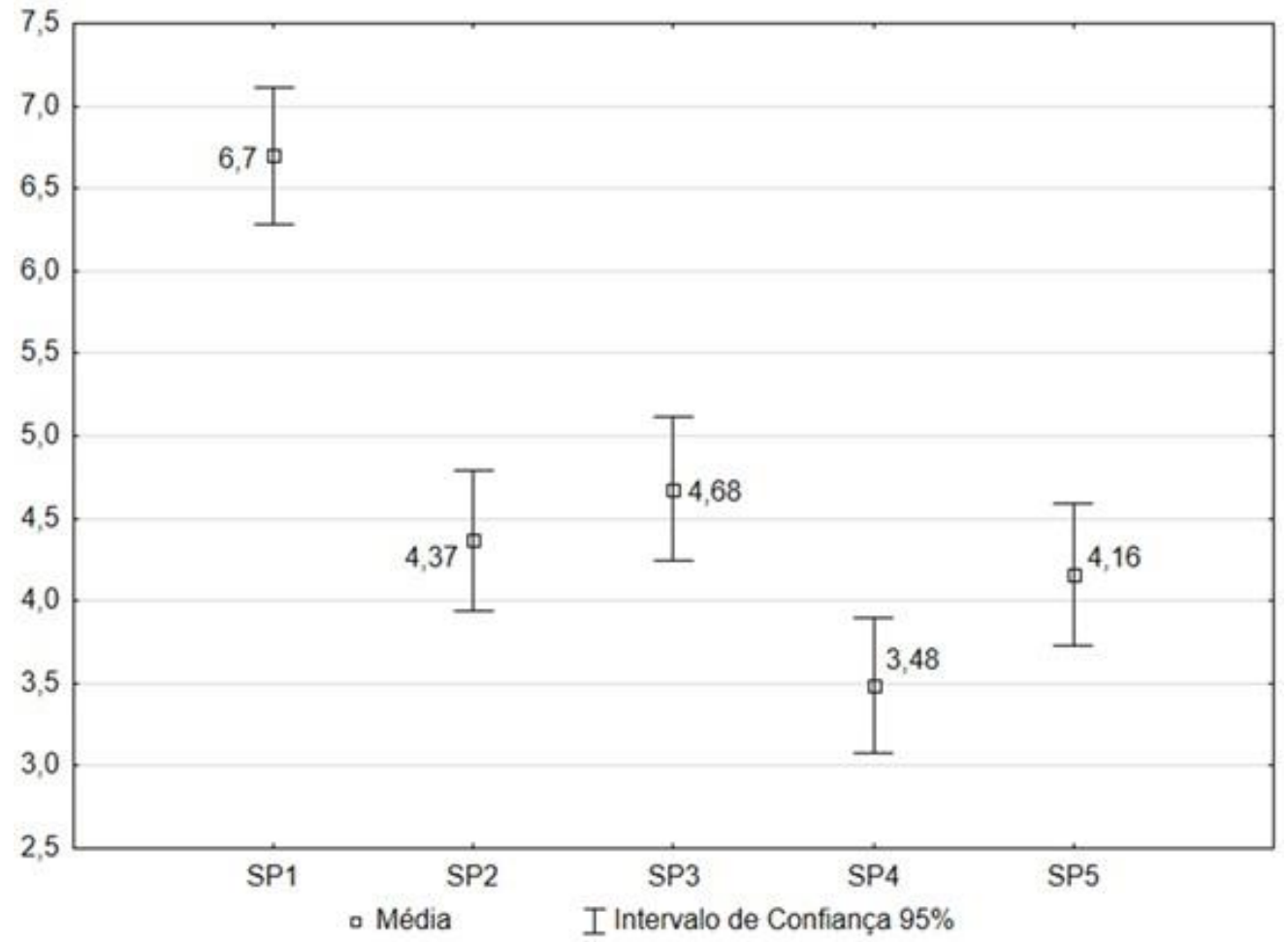

Legenda: SP - Serviços Prestados; SP1 - Orientações para manter a criança saudável; SP2 - Segurança no lar; SP3 - Mudanças no crescimento e desenvolvimento; SP4 - Maneiras de lidar com os problemas de comportamento da criança; SP5 - Maneiras para manter a criança segura.

FIGURA 2 - Média e intervalo de confiança do componente serviços prestados do atributo integralidade na avaliação do familiar/cuidador da criança, Porto Velho, Rondônia, 2017.

Dos cinco itens avaliados no componente serviços prestados, quatro itens apresentaram baixa orientação para APS, a saber: orientações sobre segurança no lar (4,37), mudanças no crescimento e desenvolvimento $(4,68)$, maneiras de lidar com os problemas de comportamento da criança $(3,48)$ e maneiras para manter a criança segura $(4,16)$. Na perspectiva dos cuidadores, o único item satisfatório foi referente ao recebimento de orientações para manter a criança saudável $(6,7)$.

\section{DISCUSSÃo}

A utilização do instrumento PCATool versão criança adaptado à realidade brasileira e validado permitiu a avaliação da presença e extensão do atributo integralidade na assistência à saúde da criança sob a ótica dos próprios familiares/cuidadores. Possibilitou também a identificação de aspectos de estrutura e processo dos serviços avaliados que necessitam de mudanças e de uma reestruturação para melhor qualificar a atenção primária no município de Porto Velho.

$\mathrm{Na}$ avaliação dos componentes do atributo integralidade, tanto o escore médio dos serviços disponíveis $(4,67)$ quanto o escore médio dos serviços prestados $(5,26)$ à criança, mostrou baixa orientação para a APS, evidenciado pela insatisfação dos responsáveis, semelhante ao encontrado em outros estudos que também obtiveram escores abaixo de 6,6 na avaliação desse atributo ${ }^{5-8,10,11}$. 
Com relação aos serviços disponíveis, o item referente à disponibilidade de imunizações teve um resultado satisfatório evidenciado pelo maior escore obtido nesse componente, o que revela o comprometimento das unidades de Estratégia de Saúde da Família (ESF) do munícipio em garantir à imunização para todas as crianças conforme o calendário vacinal nacional. Ademais, o Programa Nacional de Imunização (PNI) é uma referência internacional de política pública de saúde e exerce importante impacto na redução de doenças imunopreveníveis ${ }^{20}$.

No entanto, ainda com relação aos serviços disponíveis, dentre os itens avaliados como insatisfatórios destacaramse: a disponibilidade de programa de assistência social ou benefícios sociais, programa de suplementação nutricional, aconselhamento ou tratamento para o uso prejudicial de drogas, aconselhamento para problemas de saúde mental e sobre avaliação visual.

Tais resultados corroboram com os achados em outros estudos que mostraram esses mesmos itens como motivos para a avaliação negativa dos responsáveis pelas crianças atendidas, o que sugere a necessidade de aprimoramento das práticas assistenciais mediante melhoria na qualificação profissional e implementação de ações que sejam resolutivas frente à demanda da clientela $a^{5,6,9,11,13}$.

A partir desses estudos, observa-se que a dificuldade para o desenvolvimento de uma assistência à saúde da criança de qualidade não se encontra apenas no munícipio de Porto Velho, mas também em outras regiões do Brasil. Isso revela a presença de fragilidades na APS, sobretudo no que diz respeito à incorporação das práticas assistenciais às crianças, pautadas nos atributos essenciais da APS, visto que ações consideradas básicas essenciais como orientações e aconselhamentos, não estão sendo realizadas de forma eficaz na visão da totalidade dos familiares/cuidadores.

É crucial que os profissionais dos serviços de saúde avaliados ampliem sua atuação, sobretudo no que tange ao componente dos serviços disponíveis, considerando o contexto em que vivem as crianças e famílias, bem como os problemas mais comuns apresentados por estes ${ }^{11,12}$.

No cenário das comunidades situadas em torno das unidades de APS, os problemas relacionados ao uso de drogas e à saúde mental, podem ser recorrentes. A partir dos resultados do estudo, evidenciou-se a insatisfação dos usuários do serviço quanto à falta de aconselhamentos e orientações sobre uso de drogas e problemas de saúde mental, o que reflete a fragilidade dos serviços em abordar tais temas e junto com a população, procurar formas de enfrentamento dessas condições que muitas vezes estão presentes e influenciam o convívio familiar 5,6,8,21.

Outro fato relevante foi a baixa avaliação da população no que diz respeito à inexistência ou indisponibilidade de programas de suplementação nutricional. Isso reflete também um grande problema na atenção à saúde das crianças de Porto Velho, visto que compromete a promoção e proteção de sua saúde, pois estes programas são serviços fundamentais para o adequado crescimento e desenvolvimento infantil da região ${ }^{22}$. Isto posto, pode-se afirmar que a ineficiência ou ausência de serviços de suplementação nutricional no município pode ser um determinante para o surgimento de problemas como infecções, desnutrição, alimentação inadequada, entre outros ${ }^{23}$.

No que diz respeito ao componente serviços prestados à criança observou-se que, embora os cuidadores tenham referido satisfação com as orientações para manter a criança saudável, demonstraram uma contradição ao avaliarem negativamente os itens referentes às orientações sobre segurança no lar, mudanças no crescimento e desenvolvimento, maneiras de lidar com os problemas de comportamento da criança e maneiras para manter a criança segura. A deficiência dessas orientações indica o predomínio da lógica biologicista presente na prática assistencial dos profissionais em detrimento de um cuidado integral à criança e sua família 5,7,12,13.

Por outro lado, a dimensão orientações para manter a criança saudável, também foi o único item com escore médio satisfatório $(7,6)$ observado em unidades básicas de saúde de um município da região nordeste do país, revelando a preocupação dos profissionais em abordar os aspectos relacionados a sono, higiene e alimentação saudável no atendimento às crianças e suas famílias ${ }^{5}$.

A avaliação dos serviços prestados à criança tem relação direta com o modo como esse cuidado está sendo realizado ${ }^{2,4,11}$. Assim, urge entender como se dá o processo de assistência efetuado pelo profissional responsável pela criança e aperfeiçoar a assistência prestada a partir das fragilidades identificadas nos serviços ${ }^{4,11,21}$. A primeira barreira encontrada é a produção de um cuidado baseado no despejo de informações, no qual o profissional é o único detentor do saber, ocasionando um processo não dialógico, com isso afastando a responsabilidade do familiar cuidador $^{21}$. Somado a isso, existe uma cultura de não prevenção por parte da população, uma vez que na maioria das vezes o(a) usuário(a) busca o serviço de saúde apenas quando apresenta algum tipo de problema ${ }^{21-24}$.

A integralidade é reconhecida pelos usuários como ferramenta essencial no processo de cura e reabilitação das crianças, no entanto é nítida a insatisfação quando o atendimento prestado se baseia em ações fragmentadas e sem envolvimento com as individualidades da criança e sua família ${ }^{5-9}$. 
A falta ou a não fragilidade no estabelecimento de uma atenção integral na APS, tem como consequência o desenvolvimento de doenças preveníveis, o prolongamento de tratamentos e maiores custos para a reabilitação da saúde ${ }^{12,20,24}$. Esses acontecimentos refletem a ausência de medidas que auxiliam na prevenção da saúde da criança e também pelo fato de o familiar/cuidador apenas buscar, como dito anteriormente, atendimento quando o estado de saúde da criança apresenta alguma anormalidade ${ }^{24}$. Com isso, pode haver um comprometimento do papel da atenção primária e uma busca e supervalorização dos serviços de maior densidade tecnológica ${ }^{8}$.

Considerando que o estudo foi realizado em um hospital infantil de média e alta complexidade, mas que teve $75 \%$ de seus atendimentos decorrentes de demanda espontânea ${ }^{15}$, ou seja passíveis de resolução na APS, acredita-se que a maioria dos familiares/cuidadores não estão recebendo de maneira adequada os componentes relativos aos serviços disponíveis e prestados que caracterizam a integralidade da APS, sendo necessário buscar resolutividades para seus problemas de maneira curativa e emergencial em outros níveis de atenção, 40,21.

Nesse sentido, deve-se haver um replanejamento das medidas preventivas que contemplem de maneira integral as crianças atendidas na rede de atenção básica, além de uma capacitação para os profissionais que atuam na APS, para que suas ações sejam executadas de forma articulada com os familiares levando em consideração todo o contexto em que a criança está inserida a fim de garantir a autonomia e a continuidade do cuidado no domicílio com segurança e qualidade ${ }^{24}$.

\section{Limitações do estudo}

Entre as limitações do presente estudo, deve-se considerar o fato de os resultados obtidos expressarem a realidade específica de familiares/cuidadores abordados em uma unidade hospitalar do município, o que pode não representar a visão da totalidade de responsáveis pelas crianças cadastradas e atendidas no âmbito da APS em Porto Velho, Rondônia. O tipo de delineamento transversal desta pesquisa, também pode ser considerado como limitação, uma vez que não permitiu efetuar inferências causais na análise dos escores atribuídos pelos familiares/cuidadores ao atributo da integralidade do cuidado e analisar outros fatores com impacto na qualidade dos serviços prestados nas unidades de saúde da família. Assim, ressalta-se a necessidade de futuras pesquisas de caráter mais abrangente que permitam a generalização dos resultados e a ampliação do conhecimento sobre a temática da avaliação da saúde da criança na APS no Brasil.

\section{CONCLUSÃO}

Conclui-se que o atributo integralidade está presente na atenção à saúde da criança, nas unidades estudadas, porém de forma fragmentada, necessitando que tais serviços revejam as prioridades nos cuidados desenvolvidos junto a criança e seu familiar/cuidador.

Os baixos escores obtidos na avaliação dos familiares/cuidadores sobre os serviços disponíveis e serviços prestados às crianças nas unidades de atenção básica evidenciaram a não conformidade com o princípio de integralidade preconizado pela APS. Muitos são os desafios para uma prática de qualidade e resolutiva, sendo urgente a necessidade de rever as falhas encontradas na assistência desenvolvida nesse primeiro nível de atenção. Persiste ainda o "fazer biologicista", construído pelo modelo tradicional centrado na doença, em que as práticas profissionais podem não contemplar a totalidade das necessidades apresentadas pela demanda, o que resulta em um cuidado fragmentado.

A partir da visão dos familiares/cuidadores foi possível verificar a necessidade de um replanejamento de ações de orientação por parte da equipe profissional, para que compreendam o contexto em que a criança está inserida e que a partir dessa identificação desenvolvam ações preventivas mais eficazes, que priorizem as individualidades da criança e sua família.

Os resultados mostraram que a atenção à saúde da criança na APS no município de Porto Velho, Rondônia apresenta fragilidades, evidenciando que o cuidado não se dá de forma integral e há carência de orientações e auxílio no enfrentamento de condições que impactam a saúde da criança e o contexto familiar.

Nesse sentido, evidencia-se a necessidade de incorporação de práticas voltadas à promoção da saúde infantil, a exemplo de uma maior comunicação entre profissionais e usuários, a abordagem sobre nutrição na infância, crescimento e desenvolvimento da criança, o uso prejudicial de drogas e aconselhamento para problemas de saúde mental e avaliação para identificação de problemas visuais, já que estes foram alguns dos componentes que receberam avaliações mais negativas. Essas práticas poderão melhorar a realidade dos serviços de saúde no município, visto que muitos dos familiares/cuidadores relataram o desconhecimento sobre o leque de serviços que deveriam estar disponíveis e serem prestados nas unidades de saúde da família. 


\section{REFERÊNCIAS}

1. Ministério da Saúde. Secretaria de Atenção à Saúde. Departamento de Atenção Básica. Portaria de Consolidação $n=02$, Anexo XXII, de 28 de setembro de 2017. [site da Internet]. Aprova a Política Nacional de Atenção Básica. Brasília: Ministério da Saúde, 2017. [cited 2020 Sep 10] Available from: http://bvsms.saude.gov.br/bvs/saudelegis/gm/2017/prc0002_03_10_2017.html.

2. Starfield B. Primary Care: balancing health needs, services, and technology. Brasília: Organização das Nações Unidas para a Educação, a Ciência e a Cultura, Ministério da Saúde; 2002. [cited 2018 Jan 07]. Available from: http://bvsms.saude.gov.br/bvs/publicacoes/atencao_primaria_p1.pdf

3. Jie Mei, Yuan Liang, LeiYu Shi, JingGe Zhao, YuTan Wang, Li Kuang. The development and validation of a rapid assessment tool of primary care in China. BioMed. Res. Int. [Internet], 2016 [cited 2020 Mar 15]; 1-3. DOI: https://doi.org/10.1155/2016/6019603

4. Wang W, Shi L, Yin A, Mao Z, Maitland E, Nicholas S et al. Primary care quality among different health care structures in Tibet, China. Biomed. Res. Int. [Internet], 2015 [cited 2020 Feb 23]; (ID206709):1-8. Available from: http://downloads.hindawi.com/journals/bmri/2015/206709.pdf

5. Diniz SGM, Damasceno SS, Coutinho SED, Toso BRGO, Collet N. Evaluating comprehensiveness in children's healthcare. Rev. Gaúcha Enferm. [Internet], 2016 [cited 2020 Oct 02]; 37(4):e57067. Available from: https://www.scielo.br/pdf/rgenf/v37n4/en_0102-6933-rgenf-1983-144720160457067.pdf

6. Ferreira TLS, Costa ICC, Andrade FB. Evaluation attribute integrality in childcare services in

1. primary health care. Rev. Ciênc. Plural. [Internet], 2015 [cited 2019 Dec 26]; 1(1):22-9. Available from: https://periodicos.ufrn.br/rcp/article/view/7320/5497

7. Marques AS, Freitas DA, Leão CDA, Oliveira SKM, Pereira MM, Caldeira AP. Primary Care and maternal and child health: perceptions of caregivers in a rural 'quilombola' community. Ciênc. saúde coletiva [Internet], 2014 [cited 2020 Nov 02]; 19(2):365-71. DOI: https://doi.org/10.1590/1413-81232014192.02992013

8. Araujo Filho, ACA, Silva AN, Ribeiro MGC, Rocha SS, Andrade EMLR, Nogueira LT. Evaluation of Primary Healthcare from the perspective of child caregivers: an integrative review. Rev. esc. enferm. USP [Internet]. 2019 [cited 2020 Oct 30]; 53:e03527. DOI: https://doi.org/10.1590/s1980-220x2018030003527

9. Silva SA, Fracolli LA. Evaluating child care in the Family Health Strategy. Rev. Bras. Enferm. [Internet]. 2016 [cited 2020 Feb 28]; 69(1):54-61. DOI: http://dx.doi.org/10.1590/0034-7167.2016690107i

10. Fausto MC, Bousquat A, Lima JG. Evaluation of Brazilian Primary Health Care From the Perspective of the Users: Accessible, Continuous, and Acceptable? J. Ambul. Care Manage [Internet]. 2017 [cited 2019 Sep 28]; 40(2):S60-70. Available from: https://www.resbr.net.br/wp-content/uploads/2017/03/Evaluation_of_Brazilian_Primary_Health_Care_From.7.pdf

11. Silva SA, Baitelo TC, Fracolli LA. Primary health care evaluation: the view of clients and professionals about the family health strategy. Rev. Latino-Am. Enfermagem. [Internet]. 2015 [cited 2018 Jan 07]; 23(5):979-87. Available from: https://doi.org/10.1590/0104-1169.0489.2639

12. Harzheim Erno, Pinto Luiz Felipe, Hauser Lisiane, Soranz Daniel. Assessment of child and adult users of the degree of orientation of Primary Healthcare in the city of Rio de Janeiro, Brazil. Ciênc. saúde coletiva [Internet]. 2016 [cited 2020 Nov 01]; 21(5):1399408. DOI: https://doi.org/10.1590/1413-81232015215.26672015

13. Oliveira VBCA, Veríssimo MLOR. Children's health care assistance according to their families: a comparison between models of Primary Care. Rev. Esc. Enferm. USP [Internet]. 2015 [cited 2018 Apr 24]; 49(1):30-6. DOI: http://dx.doi.org/10.1590/S0080623420150000100004

14. Instituto Brasileiro de Geografia e Estatística (IBGE) [Internet]. Brasil, Rondônia: População. Porto Velho (RO): IBGE; 2020. [cited 2020 Oct 25]. Available from: https://cidades.ibge.gov.br/brasil/ro/porto-velho/panorama

15. Prefeitura de Porto Velho. Prefeitura do Município de Porto Velho. Secretaria Municipal de Saúde. SIPACTO. Pactuação de diretrizes, objetivos, metas e indicadores, 2015.

16. Centers for disease control and prevention - CDC [site da Internet]. EPI INFO, 2010. [cited 2018 Jul 23] Available from: https://www.cdc.gov/epiinfo/support/downloads/prevversions.html

17. Almeida C, Macinko J. Validação de uma metodologia de avaliação rápida das características organizacionais e do desempenho dos serviços de atenção básica do Sistema Único de Saúde (SUS) em nível local. Brasília: Organização Pan-Americana da Saúde; 2006.

18. Ministério da Saúde (Br). Secretaria de Vigilância em Saúde. Departamento de Vigilância Epidemiológica. PCATool-Brasil - 2020: Manual do Instrumento de Avaliação da Atenção Primária à Saúde. Brasília (DF): Ministério da Saúde; 2020. [cited 2018 Dec 4] Available from: https://www.conasems.org.br/wp-content/uploads/2020/05/Pcatool_2020.pdf

19. Ministério da Saúde (Br). Resolução no 466/12, de 12 de dezembro de 2012. [Internet]. Aprova as diretrizes e normas regulamentadoras de pesquisas envolvendo seres humanos e revoga as Resoluções CNS 196/96, 303/2000 e 404/2008. [cited 2018 Dec 4] Available from: http://bvsms.saude.gov.br/bvs/saudelegis/cns/2013/res0466_12_12_2012.html

20. Ministério da Saúde (Br). Secretaria de Vigilância em Saúde. Programa Nacional de Imunizações: coberturas vacinais no Brasil. Período: 2010-2014. Brasília (DF): Ministério da Saúde; 2015 [cited 2019 Mar 28]. Available from: https://portalarquivos2.saude.gov.br/images/pdf/2017/agosto/17/AACOBERTURAS-VACINAIS-NO-BRASIL---2010-2014.pdf

21. Damasceno SS, Nóbrega VM, Coutinho SED, Reichert APS, Toso BRGO, Collet N. Children's Health in Brazil: orienting basic network to Primary Health Care. Ciênc. Saúde Colet. [Internet]. 2016 [cited 2019 Mar 28]; 21(9):2961-73. Available from: http://www.scielo.br/pdf/csc/v21n9/1413-8123-csc-21-09-2961.pdf 
22. Governo de Rondônia. Secretaria de Estado da Saúde-SESAU: Relatório de gestão 2015. [Internet] Resultados e Perspectivas/Governo do estado de Rondônia. 2016, 438p. [cited 2018 Jun 12] Available from:

http://www.rondonia.ro.gov.br/agevisa/sobre/4-relatorio-de-gestao/

23. Ministério da Saúde (Br). Portaria no 1.130, de 5 de agosto de 2015. [site da Internet] Institui a Política Nacional de Atenção Integral à Saúde da Criança (PNAISC) no âmbito do Sistema Único de Saúde (SUS). [cited 2018 Mar 28] Available from: http://bvsms.saude.gov.br/bvs/saudelegis/gm/2015/prt1130_05_08_2015.html

24. Góes, F., \& Cabral, I. A alta hospitalar de crianças com necessidades especiais de saúde e suas diferentes dimensões [Hospital discharge in children with special health care needs and its different dimensions]. Rev. enferm. UERJ [Internet]. 2017 [cited 2020 oct 24]; 25:(e18684). DOI: https://doi.org/10.12957/reuerj.2017.18684 